\title{
SINGH'S THEOREM IN THE LATTICE CASE
}

\author{
Michael WOODROOFE * and Myoungshic JHUN ** \\ Department of Statistics, The Universtty of Michtgan, 1444 Mason Hall, Ann Arbor, MI 48109-1027, USA
}

Recerved November 1987

Revised March 1988

\begin{abstract}
The asymptotic behavior of the parametnc bootstrap estimator of the sampling distribution of a maximum likelihood estimator is investigated in a simple lattice case, integer valued random vanables whose distributions form an exponential family. The exptected value of the bootstrap estimator is compared with an Edgeworth expansion, less the contınuity correction
\end{abstract}

AMS (1980) Subject Classification· 62E20.

Keywords bootstrap estımator, Edgeworth expansıons, fractıonal part, maxımum lıkelıhood estımator, very weak expansıons.

\section{The main result}

Singh's (1981) theorem asserts that the bootstrap estimator of the distribution of a standardized sum of $n$ i.i.d. non-lattice random vanables with three moments differs from the true value by a term of smaller order of magnitude than $1 / \sqrt{n}$ w.p. 1 as $n \rightarrow \infty$. The purpose of this note is to investigate the lattice case. It is shown that the expected value of the bootstrap estimator differs from the very weak expansions of Stein (1985) and Woodroofe (1986) by a term of order $1 / \sqrt{n}$, the coefficient of which is very small in many examples.

To isolate the issues, the result is presented in a simple context. Thus, let $X_{1}, X_{2}, \ldots$ denote i.i.d. integer valued random variables with common discrete density (mass function) of the form

$f_{\omega}(x)=h(x) \exp [\omega x-\psi(\omega)], \quad x \in \mathbf{Z}, \omega \in \Omega$,

where $\mathbf{Z}$ denotes the integers, $h$ is a non-negative function and $\omega$ denotes an unknown parameter

* Research supported by the national Science Foundation. under DMS-8413452

* * Research supported by the national Science Foundation, under DMS-8702980 with values in an open interval $\Omega$. It is assumed throughout the greatest common divisor of $x-y$ for which $h(x)>0$ and $h(y)>0$ is one.

the mean and variance of $X$ are

$\theta=\psi^{\prime}(\omega)$ and $\sigma^{2}(\theta)=\psi^{\prime \prime}(\omega)$,

where ' denotes differentiation. Regarding $\sigma^{2}$ as a function of $\theta$ here is justified, since $\theta$ and $\omega$ are increasing functions of each other.

Let $\bar{X}_{n}$ denote the sample mean; $\bar{X}_{n}=S_{n} / n$, where $S_{n}=X_{1}+\cdots+X_{n}, n \geqslant 1$. Then $\bar{X}_{n}$ is the maximum likelihood estimator of $\theta$, whenever it is in $\psi^{\prime}(\Omega)$. For purposes of inference, the distributions of the (approximately) pivotal quantities

$Z_{n}=\frac{\sqrt{n}}{\sigma}\left(\bar{X}_{n}-\theta\right)=\frac{S_{n}-n \theta}{\sigma \sqrt{n}}, \quad n \geqslant 1$,

are of interest. The distribution function of $Z_{n}$ is denoted by

$G_{n}(\omega, t)=P_{\omega}\left\{Z_{n} \leqslant t\right\}$,

where $t \in \mathbb{R}, \omega \in \Omega, n \geqslant 1$. It depends on the unknown parameter $\omega$ as well as $n$.

To estimate these distributions, Efron (1979) has suggested the (parametric) bootstrap estimator

$\hat{G}_{n}(t)=G_{n}\left(\hat{\omega}_{n}, t\right), \quad t \in \mathbb{R}, n \geqslant 1$, 
where

$\hat{\omega}_{n}=\hat{\omega}_{n}\left(X_{1}, \ldots, X_{n}\right)$

denotes the maximum likelihood estımator of $\omega$ (the solution to the equation $\psi^{\prime}(\omega)=\bar{X}_{n}$, if one exists). For present purposes, it is convenient to allow $\hat{\omega}_{n}, n \geqslant 1$, to denote any strongly consistent sequence of estimators for which

$$
\begin{aligned}
\lim _{n}\left\{P_{\omega}\left\{\left|n \psi^{\prime}\left(\hat{\omega}_{n}\right)-S_{n}\right| \geqslant \varepsilon\right\}\right. \\
\left.\quad+\sqrt{n} P_{\omega}\left\{\left|\hat{\omega}_{n}-\omega\right| \geqslant \varepsilon\right\}\right\}=0
\end{aligned}
$$

for all $\varepsilon>0$ and $\omega \in \Omega$. Such a sequence may be constructed by letting $\hat{\omega}_{n}$ denote the maximum likelihood estimator for a restricted model in which $\Omega$ is replaced by a compact $\Omega_{n} \subset \Omega$ and $\Omega_{n}$ increases to $\Omega$ as $n \rightarrow \infty$.

For the related case in which the distribution of $X_{1}$ is non-lattice, (a simple variation on) Singh's (1981) Theorem shows that $\hat{G}_{n}$ and $G(\omega, \cdot)$ differ by $o(1 / \sqrt{n})$ w.p. $1\left(P_{\omega}\right)$ for all $\omega$. This is not true in the lattice case.

If $t \in \mathbb{R}$, denote its integer part by $[t]$; and let $\langle t\rangle=t-[t]$, denote the fractional part of $t$.

Proposition. If $\Omega_{0}$ is any compact subınterval of $\Omega$, then

$$
\begin{aligned}
& G_{n}(\omega, t) \\
& =\Phi(t)+\frac{1}{\sqrt{n}} \phi(t)\left[\frac{\rho}{6}\left(1-t^{2}\right)+R_{n}(\omega, t)\right] \\
& \quad+o\left(\frac{1}{\sqrt{n}}\right)
\end{aligned}
$$

uniformly in $t \in \mathbb{R}$ and $\omega \in \Omega_{0}$ as $n \rightarrow \infty$, where $\Phi$ denotes the standard normal distribution function, $\phi$ denotes the standard normal density,

$\rho=\rho(\omega)=\psi^{\prime \prime}(\omega) / \sigma^{3}$,

and

$R_{n}(\omega, t)=\frac{1}{\sigma}\left\{\frac{1}{2}-\langle n \theta+\sqrt{n} \sigma t\rangle\right\}$

for $t \in \mathbb{R}, \omega \in \Omega$, and $n \geqslant 1$.

Proof. For fixed $\omega$, this is simply Theorem 1 (p. 213) of Gnedenko and Kolmogorov (1968). The uniformity follows from an examination of the proof.
Corollary 1. For $t \in \mathbb{R}, \omega \in \Omega$, and $n \geqslant 1$, let

$G_{n}^{*}(\omega, t)=\Phi(t)+\frac{\rho}{6 \sqrt{n}}\left(1-t^{2}\right) \phi(t)$.

Then

$\lim _{n} \int_{\Omega} \sqrt{n}\left[G_{n}(\omega, t)-G_{n}^{*}(\omega, t)\right] \xi(\omega) \mathrm{d} \omega=0$

for all $t \in \mathbb{R}$ and all densities $\xi$ with compact support in $\Omega$.

Proof. It suffices to show that

$\lim _{n} \int_{\Omega}\left\{\frac{1}{2}-\langle n \theta+\sqrt{n} \sigma\rangle\right\} \xi \mathrm{d} \omega=0$

for all integrable $\xi$; and since the term in $\{\cdots\}$ is bounded, it suffices to show this when $\xi$ is the indicator of an interval $(a, b) \subset \Omega$, by a standard approximation argument. See, for example, Billingsley (1979, p. 226). Let $v(\omega)=\psi^{\prime}(\omega)$ and $v_{n}(\omega)=\psi^{\prime}(\omega)+\sqrt{\psi}^{\prime \prime}(\omega) / \sqrt{n}$ for $\omega \in \Omega$ and $n \geqslant$ 1. Then $v_{n}$ converges to $v$ and $v_{n}^{\prime}$ converges to $v^{\prime}$ uniformly on compacts as $n \rightarrow \infty$; and $v^{\prime}=\psi^{\prime \prime}$ is continuous and positive. So, if $\Omega_{0}$ is any compact subset of $\Omega$, then the restrictions of $v$ and $v_{n}$ to $\Omega_{0}$ have inverses $w$ and $w_{n}$ for which $w_{n}$ and $w_{n}^{\prime}$ converge to $w$ and $w^{\prime}$, pointwise and boundedly. If $(a, b]$ is any finite subinterval of $\Omega$, then

$$
\begin{aligned}
\int_{a}^{b} & \left\{\frac{1}{2}-\langle n \theta+\sqrt{n} \sigma\rangle\right\} \xi \mathrm{d} \omega \\
& =\int_{\alpha_{n}}^{\beta_{n}}\left\{\frac{1}{2}-\langle n y\rangle\right\} \frac{1}{w_{n}^{\prime}(y)} \mathrm{d} y,
\end{aligned}
$$

where $\alpha_{n}=v_{n}(a)$ and $\beta_{\mathrm{n}}=v_{n}(b)$ for $n \geqslant 1$. So, it suffices to show that the right side converges to zero as $n \rightarrow \infty$ for arbitrary choices of $a, b \in \Omega$. Since $w_{n}^{\prime}$ converges boundedly to $w^{\prime}$, it suffices to show this when $w_{n}^{\prime}$ is replaced by $w^{\prime}$; by the standard approximation, it suffices to show it when $1 / w^{\prime}$ is replaced by the indicator of an interval; and the latter condition is clearly satisfied.

If $G(\omega, t)$ is regarded as the coverage probability of a confidence set at $\omega$, then $\int_{\Omega} G_{n}(\omega, t)$ $\xi(\omega) \mathrm{d} \omega$ may be regarded as the long run relative frequency of converage in many independent replications of the experiment, when $\omega$ is drawn from 
the density $\xi$; so, $\int_{\Omega} G_{n}(\omega, t) \xi(\omega) \mathrm{d} \omega$ may reasonably be called the average coverage probability at $\xi$. See Woodroofe (1986) for an elaboration of this point. The bootstrap estımator is compared to $G_{n}^{*}$ below.

Theorem 1. For all $t \in \mathbb{R}$ and all $\omega \in \Omega$ for which $\rho(\omega) \neq 0$.

$$
\begin{aligned}
& \lim _{n} \sqrt{n}\left[\hat{G}_{n}(t)-G_{n}^{*}(\omega, t)\right] \\
& -\frac{1}{\sigma} \phi(T)\left\{\frac{1}{2}-\left\langle\langle t \sqrt{n} \sigma\rangle+\frac{1}{2} \rho \sigma t Z_{n}\right\rangle\right\}=0
\end{aligned}
$$

in $P_{\omega}$-probability, where $Z_{n}$ is as in (1).

Proof. Fix values of $t \in \mathbb{R}$ and $\omega \in \Omega$. Then, by the Proposition,

$$
\begin{aligned}
& \sqrt{n}\left[\hat{G}_{n}(t)-G_{n}^{*}(\omega, t)\right] \\
& =\frac{1}{6}\left(\hat{\rho}_{n}-\rho\right)\left(1-t^{2}\right) \phi(t) \\
& \quad+\phi(t) R_{n}\left(\hat{\omega}_{n}, t\right)+o(1)
\end{aligned}
$$

w.p. $1\left(P_{\omega}\right)$ as $n \rightarrow \infty$, where $\hat{\rho}_{n}=\rho\left(\hat{\omega}_{n}\right)$, for all $n \geqslant 1$. Clearly

$$
\lim _{n}\left|\frac{1}{6}\left(\hat{\rho}_{n}-\rho\right)\left(1-t^{2}\right) \phi(t)\right|=0
$$

in $P_{\omega}$-probability as $n \rightarrow \infty$, by the assumed consistency of $\hat{\omega}_{n}, n \geqslant 1$. Next, since $S_{n}$ is an integer.

$$
\begin{array}{r}
R_{n}\left(\hat{\omega}_{n}, t\right)=\hat{\sigma}_{n}^{-1}\left\{\frac{1}{2}-\left\langle\langle t \sqrt{n} \sigma\rangle+t \sqrt{n}\left(\hat{\sigma}_{n}-\sigma\right)\right.\right. \\
\left.\left.+n \hat{\theta}_{n}-S_{n}\right\rangle\right\}
\end{array}
$$

for all $n$, where $\hat{\theta}_{n}=\psi^{\prime}\left(\hat{\omega}_{n}\right)$ and $\hat{\sigma}_{n}=\sigma\left(\hat{\theta}_{n}\right)$ $=\sqrt{\psi}^{\prime \prime}\left(\hat{\omega}_{n}\right)$. By (2), a simple Taylor series approximation, and the law of the iterated logarithm

$t \sqrt{n}\left(\hat{\sigma}_{n}-\sigma\right)+n \hat{\theta}_{n}-S_{n}=\frac{1}{2} \rho \sigma t Z_{n}+o_{p}(1)$

in $\boldsymbol{P}_{\omega}$-probability as $n \rightarrow \infty$. So, since the fractional part is continuous, except for a set of content zero,

$$
\begin{aligned}
R_{n}\left(\hat{\omega}_{n}, t\right)= & \sigma^{-1}\left\{\frac{1}{2}-\left\langle\langle t \sqrt{n} \sigma\rangle+\frac{1}{2} \rho \sigma t Z_{n}\right\rangle\right\} \\
& +o_{p}(1)
\end{aligned}
$$

under $P_{\omega}$ as $n \rightarrow \infty$. See the lemma below with $U_{n}=\langle t \sqrt{n} \sigma\rangle+t \sqrt{n}\left(\hat{\sigma}_{n}-\sigma\right)+n \hat{\theta}_{n}-S_{n}$

and

$V_{n}=\langle t \sqrt{n} \sigma\rangle+\frac{1}{2} \rho \sigma t Z_{n}$.

The theorem follows by collecting terms.
Corollary 2. For $r>0$ and $0 \leqslant m<1$, let $e(m, r)=\int_{\mathbb{R}}\left[\frac{1}{2}-\langle m+r s\rangle\right] \phi(s) \mathrm{d} s$.

Then

$$
\begin{aligned}
E_{\omega} & \left\{\hat{G}_{n}(t)-G_{n}^{*}(\omega, t)\right\} \\
& =\frac{1}{\sigma \sqrt{n}} \phi(t) e\left(\langle t \sqrt{n} \sigma\rangle, \frac{1}{2} \rho \sigma t\right)+\mathrm{o}\left(\frac{1}{\sqrt{n}}\right)
\end{aligned}
$$

as $n \rightarrow \infty$ for all $t \in \mathbb{R}$ and all $\omega \in \Omega$ for which $\rho(\omega) \neq 0$.

Proof. If $\omega \in \Omega$, then there is a compact $\Omega_{0} \subset \Omega$, for which $P_{\omega}\left\{\hat{\omega}_{n} \notin \Omega_{0}\right\}=\mathrm{o}(1 / \sqrt{n})$ as $n \rightarrow \infty$, by (2). It is clear that

$$
\left(\hat{\rho}_{n}-\rho\right) I\left\{\hat{\omega}_{n} \in \Omega_{0}\right\}, \quad n \geqslant 1,
$$

and

$\left(1 / \hat{\sigma}_{n}\right) R_{n}\left(\hat{\omega}_{n}, t\right) I\left\{\hat{\omega}_{n} \in \Omega_{0}\right\}, \quad n \geqslant 1$,

are uniformly integrable random variables. It follows that

$$
\begin{aligned}
& \lim _{n} E_{\omega}\left\{\left(\hat{\rho}_{n}-\rho\right) I\left\{\hat{\omega}_{n} \in \Omega_{0}\right\}\right\}=0 . \\
& \lim _{n} E_{\omega}\left\{\left(1 / \hat{\sigma}_{n}\right) R_{n}\left(\hat{\omega}_{n}, t\right) I\left\{\hat{\omega}_{n} \in \Omega_{0}\right\}\right\} \\
& -\frac{1}{\sigma} \phi(t) e\left(\langle t \sqrt{n} \sigma\rangle, \frac{1}{2} \rho \sigma t\right)=0 .
\end{aligned}
$$

(For the latter assertion, it is convenient to consider subsequences along which $\langle t \sqrt{n} \sigma\rangle$ is convergent.) the corollary follows easily.

Remark. The function $e$ decreases quickly as $\mathrm{r}$ increaes. To see why, write

$$
\frac{1}{2}-\langle t\rangle=\sum_{k=1}^{\infty} \frac{1}{k \pi} \sin (2 \pi k t), \quad t \in \mathbb{R} .
$$

Then

$$
\begin{aligned}
e(m, r) & =\sum_{k=1}^{\infty} \frac{1}{k \pi} \int_{\mathbf{R}} \sin (2 \pi k t) \frac{1}{r} \phi\left(\frac{t-m}{r}\right) \mathrm{d} t \\
& =\sum_{k=1}^{\infty} \frac{1}{k \pi} \operatorname{Im}\left\{\exp \left(2 \pi \mathrm{i} m k-2 r^{2} \pi^{2} k^{2}\right)\right\} \\
& =\sum_{k=1}^{\infty} \frac{1}{k \pi} \sin (2 \pi m k) \mathrm{e}^{-2 r^{2} \pi^{2} k^{2}}
\end{aligned}
$$


and for $0 \leqslant m<1$ and $r>0$. For example, $\sup _{m}\left|e\left(m, \frac{1}{2}\right)\right|<0.01, \sup _{m}|e(m, 1)|<10^{-9}$.

In cases where $\sup _{m}|e(m, \rho \sigma / 2)|$ is small, the theorem suggests that bootstrap approximations should be close to the very weak expansions cited above. This is a weaker property than that asserted in Singh's Theorem, but provides some comfort.

Example. If $X_{1}$ has the Poisson distribution with unknown mean $\theta>0$, and if $t=2$ (as in an upper $97.5 \%$ confidence bound), then $r=\rho \sigma t / 2=1$ for all $\omega$, so that the coefficient to $1 / \sqrt{n}$ is less than $10^{-9}$.

The following lemma completes the justification of (3).

Lemma. Let $U_{n}, n \geqslant 1$, and $V_{n}, n \geqslant 1$, denote random variables for which:

(1) the distrubitons of $V_{n}, n \geqslant 1$, are tight;

(ii) all weak limit points of the distributions of $V_{n}, n \geqslant 1$, assign measure zero to $\mathbf{Z}$; and

Then

(iii) $U_{n}-V_{n} \rightarrow 0$ in probability as $n \rightarrow \infty$.

$\left\langle U_{n}\right\rangle-\left\langle V_{n}\right\rangle \rightarrow 0 \quad$ in probablity.

Proof. Let $\mathbb{Z}_{\delta}$ denote the set of $x$ whose distance from $\mathbf{Z}$ is at most $\delta$. Then $\mathbf{Z}_{\delta}$ is closed, and $\mathbb{Z}_{\delta}$ decreases to $\mathbb{Z}$ as $\delta$ decreases to zero. So,

$\lim _{\sigma \rightarrow 0} \lim _{n} \sup P\left\{V_{n} \in \mathbb{Z}_{\delta}\right\}=0$,

by Theorem 2.1 of Billingsely (1968, pp. 11-12) and conditions (i) and (ii) above. Thus, given $\varepsilon>0$, there are $n_{0}$ and $\delta<\varepsilon$ for which $P\left\{V_{n} \in\right.$ $\left.\mathbb{Z}_{2 \delta}\right\} \leqslant \varepsilon / 2$ for all $n \geqslant n_{0}$. So,

$$
\begin{aligned}
& P\left\{\left|\left\langle U_{n}\right\rangle-\left\langle V_{n}\right\rangle\right| \geqslant \varepsilon\right\} \\
& \quad \leqslant P\left\{\left|U_{n}-V_{n}\right| \geqslant \delta\right\}+P\left\{V_{n} \in \mathbf{Z}_{2 \delta}\right\} .
\end{aligned}
$$

for all $n \geqslant n_{0}$; and this is less than $\varepsilon$ for all sufficiently large $n$.

\section{A Studentized version}

In this section, suppose that $\hat{\omega}_{n}$ satisfies the more stringent condition

$\lim _{n} \sqrt{n} P_{\omega}\left\{\hat{\theta}_{n} \neq \bar{X}_{n}\right\}=0, \quad \forall \omega$.
Let

$T_{n}=\left(\sqrt{n} / \hat{\sigma}_{n}\right)\left(\bar{X}_{n}-\theta\right)$

and

$H_{n}(\omega, t)=P_{\omega}\left\{T_{n} \leqslant t\right\}$

for all $t \in \mathbb{R}, \omega \in \Omega$, and $n \geqslant 1$. Then an expansion for $H_{n}$ may be obtained as follows. For fixed $\omega$, let $h(x)=h_{\omega}(x)=(x-\theta) / \sigma(x)$, for $x \in$ $\psi^{\prime}(\Omega)$. Then it is easily seen that $h$ is increasing near $x=\theta$, so that the restriction of $h$ to a suitable neighborhood of $\theta$ has an inverse function $g=g_{\omega}=h_{\omega}^{-1}$. Moreover, it is also easily seen that $g(0)=\theta, g^{\prime}(0)=\sigma \quad$ and $g^{\prime \prime}(0)=\rho \sigma$. For fixed $t \in \mathbb{R}$ and $\omega \in \Omega$, let

$t_{n}=t_{n}(\omega, t)=\frac{\sqrt{n}}{\sigma}\left[g\left(\frac{t}{\sqrt{n}}\right)-g(0)\right]$.

Then, $t_{n}=t+\rho t^{2} / 2 \sqrt{n}+o(1 / \sqrt{n})$ as $n \rightarrow \infty$; and, by ( $\left.2^{\prime}\right)$ and Chebyshev's Inequality,

$$
\begin{aligned}
& H_{n}(\omega, t) \\
&= G_{n}\left(\omega, t_{n}\right)+o(1 / \sqrt{n}) \\
&= \Phi(t)+\frac{1}{\sqrt{n}} \phi(t)\left[\frac{\rho}{6}\left(1+2 t^{2}\right)+R_{n}\left(\omega, t_{n}\right)\right] \\
&+o\left(\frac{1}{\sqrt{n}}\right)
\end{aligned}
$$

for each $t$ and $\omega$. In fact, (4) holds uniformly on compacts with respect to $t \in \mathbb{R}$ and $\omega \in \Omega$. Let

$\hat{H}_{n}(t)=h_{n}\left(\hat{\omega}_{n}, t\right)$

and

$H_{n}^{*}(\omega, t)=\Phi(t)+\frac{\rho}{6 \sqrt{n}}\left(1+2 t^{2}\right) \phi(t)$

for $t \in \mathbb{R}, \omega \in \Omega$, and $n \geqslant 1$.

Theorem 2. With the notation of the previous paragraph,

$$
\begin{aligned}
& \lim _{n} \sqrt{n}\left[\hat{H}_{n}(t)-H_{n}^{*}(\omega, t)\right] \\
& -\frac{1}{\sigma} \phi(t)\left\{\frac{1}{2}-\left\langle\left\langle\sqrt{n} \rho \sigma t^{2}\right\rangle+\frac{1}{2} \rho \sigma t Z_{n}\right\rangle\right\}=0
\end{aligned}
$$

In $P_{\omega}$-probability for each $t \in \mathbb{R}$ and $\omega \in \Omega$ for which $\rho(\omega) \neq 0$. 
Proof. The proof is similar to that of Theorem 1, but slightly more complicated, since $\hat{t}_{n}:=t_{n}\left(\hat{\omega}_{n}, t\right)$ depends on $n$ and $X_{1}, \ldots, X_{n}$. In the analysis, one finds that (with probability approaching one),

$\left\langle\sqrt{n} \hat{\sigma}_{n} \hat{t}_{n}\right\rangle=\left\langle V_{n}+o_{p}(1)\right\rangle$,

where

$V_{n}=\left\langle\sqrt{n} \sigma t+\frac{1}{2} \rho \sigma t^{2}\right\rangle+\frac{1}{2} t \rho \sigma Z_{n}, \quad n \geqslant 1$.

Here $V_{n}, n \geqslant 1$, satisfies the conditions of the lemma; and the argument proceeds as above.

\section{Acknowledgement}

Thanks to Bob Keener for helpful discussions.

\section{References}

Bıllingsley, P. (1968), Convergence of Probability Measures (W1ley, New York).

Billıngsley, P. (1979), Probabtlity and Measure (Wiley, New York).

Efron, B (1979), Bootstrap methods. Another look at the Jackknife, Ann Statıst 7, 1-26

Gnedenko, B and A. Kolmogorov (1968), Limit theorems for sums of independent random variables (Addison-Wesley, Reading, MA)

Singh, K (1981), On the asymptotic accuracy of Efron's bootstrap, Ann Statust 9, 1187-1195.

Stein, C. (1985), On the coverage probability of confidence sets based on a prior distribution, Banach Center Publications $16,485-514$

Woodroofe, M. (1986), Very weak expansions for sequentıal confidence levels, Ann Statist 14, 1049-1067 